Abstracta Iranica Abstracta Iranica

Revue bibliographique pour le domaine irano-aryen

Volume 40-41 | 2019

Comptes rendus des publications de 2017-2018

\title{
R. Todd Godwin. Persian Christians at the Chinese Court: The Xi'an Stele and the Early Medieval Church of the East
}

Florence Jullien

\section{(2) OpenEdition \\ Journals}

Édition électronique

URL : http://journals.openedition.org/abstractairanica/50948

DOI : 10.4000/abstractairanica.50948

ISBN : 1961-960X

ISSN : 1961-960X

Éditeur :

CNRS (UMR 7528 Mondes iraniens et indiens), Éditions de l'IFRI

Référence électronique

Florence Jullien, « R. Todd Godwin. Persian Christians at the Chinese Court: The Xi'an Stele and the Early Medieval Church of the East », Abstracta Iranica [En ligne], Volume 40-41 | 2019, document 15, mis en ligne le 30 décembre 2019, consulté le 20 avril 2021. URL : http://journals.openedition.org/ abstractairanica/50948 ; DOI : https://doi.org/10.4000/abstractairanica.50948

Ce document a été généré automatiquement le 20 avril 2021.

Tous droits réservés 


\title{
R. Todd Godwin. Persian Christians at the Chinese Court: The Xi'an Stele and the Early Medieval Church of the East
}

\author{
Florence Jullien
}

\section{RÉFÉRENCE}

R. Todd Godwin. Persian Christians at the Chinese Court: The Xi'an Stele and the Early Medieval Church of the East. (Library of Medieval Studies), London: I.B. Tauris, 2018, 320 p. ISBN 9781786723161

La présente étude est le fruit d'une thèse de doctorat soutenue sous la direction d'Erica C.D. Hunter dans le cadre de la School of African and Oriental Studies de l'Université de Londres. L'auteur reprend à nouveaux frais l'étude du dossier de la stèle bilingue de Xi' an dans le contexte d'une histoire générale des échanges entre la Chine des Tang, le monde syriaque et les milieux iraniens. L'inscription bilingue sur le monument érigé dans la capitale chinoise en 781 atteste en syriaque et en chinois l'existence de communautés chrétiennes dans le nord de la Chine. Depuis les premiers commentateurs jésuites du XVII ${ }^{\mathrm{e}}$ siècle jusqu'à Paul Pelliot, l'interprétation de cette inscription a été faite de façon exclusivement centrée sur le contexte culturel et historique chinois; par là-même, elle fut comprise de façon erronée dans une perspective missionnaire, comme le témoignage d'une entreprise de christianisation de la Chine focalisée sur les trois grandes religions bouddhiste, confucianiste et taoïste. Michel Tardieu (Collège de France) et Jacques Gernet (Collège de France et Académie des Inscriptions et Belles-Lettres) avaient magistralement démontré dans deux études complémentaires parues en 2008 comment le contexte théologique syro-oriental d'une part, et historique chinois d'autre part, pouvaient conduire à une nouvelle lecture des modes d'implantation de la communauté chrétienne en Chine. M. Tardieu, « Le schème hérésiologique de désignation des adversaires dans l'inscription nestorienne chinoise de Xi'an ", dans C. Jullien (ed.), Controverses des chrétiens dans l'Iran sassanide, (Studia 
Iranica. Cahier 36), Paris, 2008, p. 207-226 ; J. Gernet, « Remarques sur le contexte chinois de l'inscription de la stèle nestorienne de Xi'an", ibid., p. 227-243. Une mise en parallèle de la liste des quatre hérésies qui figurent dans le prologue de l'inscription avec les exposés de théologie syro-orientale de la même époque a permis à $M$. Tardieu de restituer une formulation traditionnelle de l'apologétique syro-orientale que l'on retrouve par exemple dans les mises en garde des synodes de l'Église de Perse entre les $\mathrm{V}^{\mathrm{e}}$ et $\mathrm{VII}^{\mathrm{e}}$ siècles. Une prise en compte du contexte chinois et une relecture soigneuse de l'inscription viennent à l'appui de cette lecture puisque J. Gernet a pu montrer que les fondations des monastères "nestoriens" à partir de 638 a été le résultat d'accords diplomatiques en faveur des communautés syro-orientales établies en Chine, dont le clergé était composé de religieux probablement originaires de Sogdiane qui ne se souciaient pas d'évangélisation. " $\mathrm{Si}$, dans sa savante traduction de cette inscription, Paul Pelliot s'est efforcé de christianiser de nombreux termes qui ne l'exigeaient pas, c'était pour se conformer à une tradition qui, depuis le XVII ${ }^{\mathrm{e}}$ siècle, n'avait jamais été remise en question. " L'A. ne tient pas compte de cette publication pourtant majeure pour la problématique abordée. Mais son traitement plus global de la documentation lui permet de reconstruire les liens complexes qui existaient entre l'Église d'Orient, la culture aristocratique sassanide et l'empire des Tang (617-907), depuis la chute de l'empire sassanide vers 651 jusqu'à l'implantation du califat abbasside.

2 Une première partie comprend deux chapitres : dans le premier (« The Late Sasanian Court and Divine Economy ", p. 31-68), l'A. examine dans une démarche comparative très stimulante le thème de la théologie politique à travers les liens entre le politique et le religieux en contexte sassanide. Le second (" 'Repairing the Imperial Net' before the An Lushan Rebellion », p. 69-104) est consacré au contexte des relations entre les empires. La seconde partie est construite sur un modèle similaire de balancement entre les deux contextes, iranien et chinois; pour chaque chapitre, la perspective comparatiste est privilégiée. Dans le troisième chapitre ("The Habitus of Patriarch Timothy I ", p. 105-138), l'A. évalue le rôle de la noblesse et des élites lettrées dans leurs relations avec les milieux de pouvoir. Le quatrième et dernier chapitre (" The Court of Emperor Tang Dezong as 'Imperial Net', and the Church of the East's Persian Longue Durée », p. 139-188) est consacré à la cour des Tang sous le règne de l'empereur Dezong (742-805) ; la terminologie braudelienne un peu étrange d'une "longue durée perse » est explicitée p. 20 : elle fait référence à la permanence de structures et d'institutions développées sous le règne des dynastes sassanides tardifs, dont le fonctionnement se prolongea durant les décennies suivantes dans des contextes politiques différents. Sur l'impact nuancé du renforcement des activités missionnaires et de la multiplication des établissements scholastiques et monastiques à la fin de l'empire sassanide, voir l'avis nuancé de V. Berti dans son étude Vita e studi di Timoteo I, patriarca cristiano di Baghdad. Ricerche sull'epistolario e sulle fonti contigue, (Studia Iranica. Cahier 41. "Chrétiens en terre d'Iran" III), Louvain, 2009. La politique de Timothée, durant une période troublée, semble surtout avoir garanti aux communautés chrétiennes centre-asiatiques la continuité de leur lien avec l'Église-mère de Mésopotamie. Deux appendices fournissent des instruments utiles: listes des rois et gouvernants perses, arabes, chinois et des catholicoi de l'Église syro-orientale (p. 195-198); inscription de la stèle en caractères chinois (p. 199-202). Deux autres sont des réflexions sur les fondements de la propagation de la religion manichéenne en Chine (p. 203-212), et sur les définitions des concepts théorétiques utilisés dans l'étude (p. 213-218). Un index réunissant à la fois les noms propres, les toponymes et des notions clôt cette étude (p. 303-306). 
3 Certaines expressions en caractères syriaques sont typographiquement trop condensées et les mots mis bout à bout sans espaces intermédiaires (par ex. p. 63). La stèle de Xi' an a été découverte en 1623, déterrée avant d'être déplacée entre 1623 et 1625 (corriger p. 10). On regrettera que la très brève histoire de la recherche (p. 18-19) ne tienne compte que des dix dernières années et n'ait pas été plus approfondie. La localisation de Veh-Ardashir sur la carte (Map 1, p.xiii) est erronée (à mettre en Asorestān). Noter également que la notion de "route de la soie" comme voie unique de transit reste discutée (Map 2, p. xiv).

4 Avec compétence, l'A. propose une nouvelle analyse de la stèle de Xi' an dont il faut souligner la qualité, qui prend en compte les sources historiques afférentes en syriaque, en grec, en arabe et en chinois. Il en ressort un panorama des échanges entre Moyen et Extrême-Orient et du développement de différentes traditions religieuses (bouddhisme, zoroastrisme, manichéisme, christianisme) au sein d'un milieu cosmopolite et ouvert aux relations interculturelles.

\section{AUTEURS}

\section{FLORENCE JULLIEN}

CNRS, Mondes iranien et indien, Paris 